\title{
Blefaroplastia associada a enxertia de pele autólogia para xantelasmas extensos: relato de caso
}

\author{
Extensive xanthelasma - a surgical solution: case report
}

\author{
Filipe José Pereira ${ }^{1}$ \\ Antonio Augusto Velasco e Cruz ${ }^{2}$ \\ Heriberto Pinto Guimarães Neto ${ }^{3}$ \\ Cristiano Coelho Ludvig'4
}

\begin{tabular}{|c|}
\hline RESUMO \\
\hline O xantelasma pode ser tratado por uso de ácidos, laser ou cirurgia com \\
fechamento primário, todos apresentando recidivas freqüentes, além de \\
ineficazes em casos extensos. Os autores apresentam a reabilitação funcio- \\
nal e estética em uma paciente com recidiva de xantelasmas extensos nas \\
porções mediais das quatro pálpebras em que foi realizada exérese das lesões \\
associadas à blefaroplastia superior e inferior, com utilização da pele retirada \\
para enxerto em transferência mútua, ou seja, o excesso de pele de pálpebras \\
superiores utilizado para enxertia em pálpebras inferiores e vice-versa. \\
\hline
\end{tabular}

Descritores: Blefaroplastia; Xantomatose/cirurgia; Doenças palpebrais/cirurgia; Procedimentos cirúrgicos reconstrutivos; Procedimentos cirúrgicos oftalmológicos; Recidiva; Transplante de pele/métodos; Relatos de casos [Tipo de publicação]

\section{INTRODUCÃO}

A palavra xantelasma origina-se do grego e significa lâmina amarelada, é também referido como xantoma palpebral, e caracteriza-se por acúmulos focais na derme de tecido conectivo fibroproliferativo associado a histiócitos repletos de $\operatorname{lipídios}^{(1-2)}$. A lesão tipicamente se apresenta como placas amareladas e amolecidas na porção medial das pálpebras, com o diagnóstico estabelecido através do exame clínico isoladamente. É uma desordem freqüente a partir dos 40 anos e com incidência discretamente maior no sexo feminino ${ }^{(3)}$.

O surgimento do xantelasma pode servir de alerta para doenças ocultas como hiperlipidemia e dislipoproteinemia familiar ${ }^{(4)}$. Trata-se de um tema controverso, pois alguns artigos referem não haver relação com hipercolesterolemia e se tratar de uma manifestação idiopática ${ }^{(5-6)}$; em contrapartida, outros artigos recomendam solicitar o perfil lipídico do paciente em virtude do diagnóstico de hiperlipidemia acometer cerca de metade dos pacientes com xantelasma ${ }^{(7-8)}$.

Apesar de raros, há relatos de regressão das lesões com a restrição dietética de gordura, após tratamento de doenças como hipotireoidismo e na reposição hormonal em mulheres no climatério ${ }^{(9-10)}$.

Em geral, há necessidade de tratamento diretamente sobre o xantelasma, entre os métodos disponíveis incluem-se o uso tópico de ácido tricloroacético, ablação a laser e excisão cirúrgica ${ }^{(11)}$. Importante ressaltar que as recidivas tumorais são freqüentes independentemente da técnica escolhida ${ }^{(4)}$.

Na maioria das vezes, o tratamento para este tipo de lesão está baseado em fatores estéticos, pois apenas em raros casos as tumorações se magnificam o bastante a ponto de obstruir o eixo visual ou de acometer grandes extensões palpebrais ${ }^{(12)}$. Nestes casos, os diversos tratamentos propostos na literatura são ineficazes ou são necessárias grandes ressecções, com prejuízo da dinâmica palpebral e estética. 
Neste âmbito, o presente trabalho tem como objetivo relatar um caso de xantelasma extenso, acometendo as quatro pálpebras de um paciente e propor técnica alternativa de reconstrução destes casos de difícil manejo.

\section{RELATO DE CASO}

A.A.C.L, 53 anos, queixava-se de lesões amareladas nas pálpebras superiores e inferiores. Ao exame, foram evidenciadas lesões amareladas, amolecidas, bastante elevadas e extensas da porção medial das pálpebras superiores e inferiores compatíveis com xantelasma (Figura 1).

Relatava excisão cirúrgica de lesões semelhantes, mas de menor tamanho, há 10 anos, com recidiva cerca de 6 meses após cirurgia.

Foi realizado exame oftalmológico completo: sem particularidades.

O rastreamento para dislipidemia evidenciou valores fora da faixa de normalidade, a seguir com os valores de referência entre parênteses: colesterol total $366 \mathrm{mg} / \mathrm{dl}$ ( $<200 \mathrm{mg} / \mathrm{dl}) ; \mathrm{HDL} 37 \mathrm{mg} / \mathrm{dl}$ (>40 mg/dl); LDL $125 \mathrm{mg} / \mathrm{dl}$ (<100 mg/dl); TGL $243 \mathrm{mg} / \mathrm{dl}$ $(<150 \mathrm{mg} / \mathrm{dl})^{(13)}$.

Não havia sinais de histiocitose sistêmica ou orbitária ${ }^{(14)}$.

Após controle clínico da dislipidemia, a paciente foi submetida à cirurgia, aqui descrita:

- Anestesia local de pálpebras superiores e inferiores com neocaína a $0,5 \%$ com adrenalina a 1: 200.000;

- Exérese de xantelasmas superiores e inferiores;

- Blefaroplastia superior e inferior em continuidade com incisão anterior lateralmente;

- Utilização da pele retirada para enxertia em transferência mútua, ou seja, o excesso de pele das pálpebras superiores utilizado para enxertia em pálpebras inferiores e vice-versa, com sutura simples com mononylon 6-0, conforme foto abaixo do pós-operatório de 1 semana (Figura 2).

Não houve intercorrência no pós-operatório, apresentando no $6^{\circ}$ mês de pós-operatório um excelente resultado funcional e estético (Figura 3).

\section{DISCUSSÃO}

São diversas as modalidades de tratamentos proposta na literatura para xantelasma, e, ao contrário de outras patologias com diversos tratamentos propostos, muitos apresentam bons resultados $^{(4)}$.

Alguns artigos recomendam a solicitação do perfil lipídi$\mathrm{co}^{(4,7-8)}$ pois seu desequilíbrio acomete cerca de metade dos pacientes com xantelasma, que no nosso relato de caso justificou esta preocupação devido aos valores fora da faixa de normalidade; apesar de seu controle não ter repercutido na extensão do xantelasma.

A literatura dermatológica relata o uso de ácido dicloroacético e tricloroacético como métodos seguros e de fácil apli-

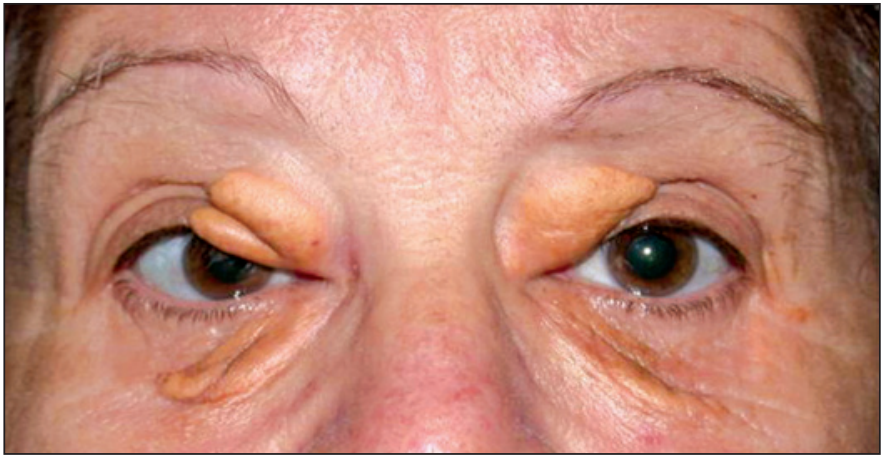

Figura 1 - Xantelasmas extensos nas quatro pálpebras: pré-operatório

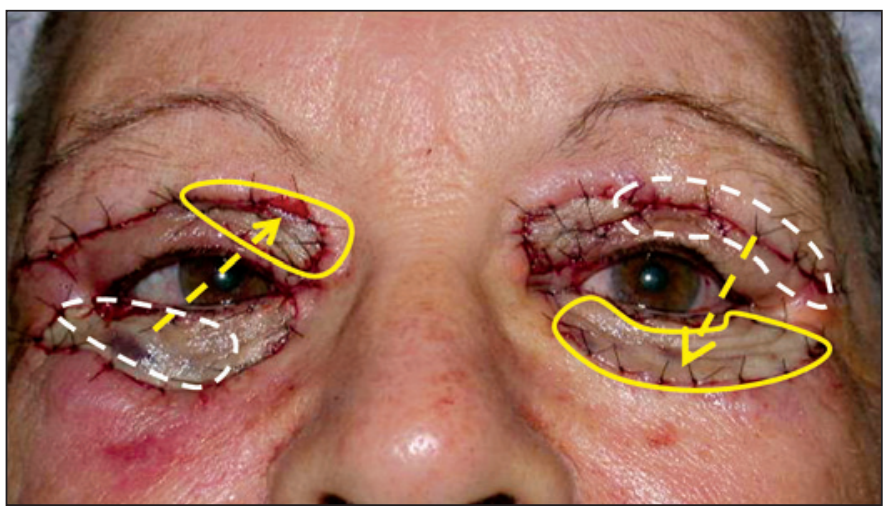

Figura 2 - Sétimo dia de pós-operatório, enxertos de pálpebras superiores em pálpebras inferiores (e vice-versa) com ótimo aspecto

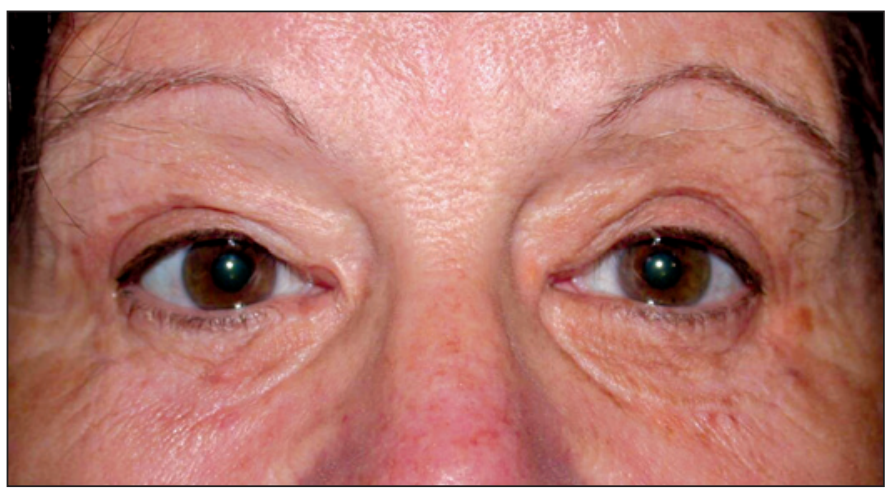

Figura 3 - Sexto mês de pós-operatório

cação ${ }^{(11)}$. A técnica consiste na aplicação tópica do ácido nas margens da lesão, com posterior neutralização com bicarbonato de sódio. O procedimento é prático, rápido e eficaz, contudo, pode ocorrer cicatrização exuberante e retração de pele causando ectrópio quando realizado nas pálpebras inferiores e lagoftalmo quando realizado nas pálpebras superiores ${ }^{(15)}$.

Ainda é grande o número de trabalhos que abordam o tratamento através da ablação com laser. O YAG-laser proporciona bons resultados para lesões superficiais, inclusive próximas à margem palpebral. O laser de dióxido de carbono é mais indica- 
do para lesões profundas por apresentar melhor hemostasia, mas está relacionado com surgimento de discromia em cerca de $10 \%$ dos casos. Há ainda a opção do laser de $\operatorname{argônio~}^{(16-18)}$.

Lesões mais extensas, acometendo pálpebras superiores e inferiores, pouco estéticas, que causam baixa auto-estima do paciente e privação social, são casos desafiadores. Impõe-se a necessidade de excisão cirúrgica ampla. Dependendo do tamanho e localização da lesão, esta pode ser incluída na incisão da blefaroplastia, ou esta ser modificada para tal ${ }^{(4)}$.

Alguns autores propõem a excisão cirúrgica das lesões, seguida de cicatrização por segunda intenção ${ }^{(19)}$, enquanto outros optam pela inclusão das lesões nas incisões clássicas de blefaroplastia. Em casos de múltiplas lesões relatam excisão cirúrgica em vários tempos, com extirpação de 1 ou 2 lesões por procedimento e intervalo de 2 meses entre um e outro ${ }^{(20)}$. Pode ser realizada uma incisão de blefaroplastia modificada capaz de incluir de maneira mais adequada a área cantal medial, topografia clássica do xantelasma ${ }^{(21)}$. Outra alternativa proposta na literatura seria a confecção de "flaps-músculo" cutâneos, preservando tecido da pálpebra superior para utilizar em caso de recorrência, como a técnica descrita por Hosokawa et al. ${ }^{(22)}$ onde o "flap" é confeccionado a partir do tecido lateral da pálpebra superior.

Como último recurso, nos casos extensos, os enxertos completam o arsenal terapêutico para reconstrução da área cruenta resultante da exérese de qualquer tumoração palpebral, com diversas opções para sítio doador, preferencialmente pele da própria pálpebra ${ }^{(23)}$.

Outro ponto relevante durante o planejamento cirúrgico é o prognóstico, pois a recorrência é comum independente da técnica escolhida ${ }^{(4,20)}$. Literatura relata $40 \%$ de recorrência após primeira excisão e $60 \%$ após a segunda ${ }^{(24)}$. No caso em estudo, a recidiva após a primeira cirurgia realizada há 10 anos, ocorreu no primeiro ano, dado que corrobora com a literatura, mas após ressecção realizada, não houve recorrência de nenhuma lesão até o momento, pós-operatório tardio de 3 anos.

Portanto, no caso de xantelasmas muito grandes, a reabilitação funcional e estética não é possível através de ressecção com fechamento primário, e neste trabalho apresentamos uma opção terapêutica com ótimo resultado para esses casos desafiadores: "blefaroplastia associada a enxertia de pele autóloga para xantelasmas extensos".

\section{ABSTRACT}

Xanthelasma can be treated with acids, laser or primary suture, all presenting frequent relapses besides being inefficacious in extensive cases. The authors present the functional and esthetic result of a female patient's case with extensive xanthelasmas on the upper and lower eyelids which were removed combined with upper and lower blepharoplasty, using the skin removed from the upper eyelids to cover the defect in the lower, and vice-versa.

Keywords: Blepharoplasty; Xanthomatosis/surgery; Eyelid diseases/surgery; Reconstructive surgical procedures; Ophthalmologic surgical procedures; Recurrence; Skin transplantation/ methods; Case reports [Publication type]

\section{REFER̂̂NCIAS}

1. Stedman's Medical Dictionary. 26 ${ }^{\text {th }}$ ed. Baltimore: Williams \& Wilkins; 1995. p.159, 828, 1170-422.

2. Cohen PR. Xanthelasma palpebrarum. J Gt Houst Dent Soc. 1996;67(9):10-1.

3. Jónsson A, Sigfusson N. Letter: Significance of xanthelasma palpebrarum in the normal population. Lancet. 1976;1(7955):372.

4. Rohrich RJ, Janis JE, Pownell PH. Xanthelasma palpebrarum: a review and current management principles. Plast Reconstr Surg. 2002;110(5):1310-4.

5. Bergdorf WH. The histiocytes. In: Elder D, editor. Lever's histopathology of the skin. $8^{\text {th }}$ ed. Philadelphia: Lippincott-Raven; c1997.

6. Older JJ. Eyelid tumors clinical diagnosis and surgical treatment. New York: Raven Press; c1987. p.43-5.

7. Goldstein JL, Brown MS. Familial hypercholesterolemia. In: Stanbury JB, Wyngaarden JB, Frederickson DS, editors. The metabolic basis of inherited disease. $6^{\text {th }}$ ed. New York: McGraw-Hill; c1989.

8. Bergman R. The pathogenesis and clinical significance of xanthelasma palpebrarum. J Am Acad Dermatol. 1994;30(2 Pt 1): 236-42.

9. Vinger PF, Sachs BA. Ocular manifestations of hyperlipoproteinemia. Am J Ophthalmol. 1970;70(4):563-73.

10. Caplan RM, Curtis AC. Xanthoma of the skin. Clinical characteristics in relation to disorders of lipid metabolism, and presentation of previously unreported cause for secondary hyperlipemic xanthomas. JAMA. 1961;176:859-64.

11. Moura EM, Volpini M. Quimioesfoliação do xantelasma palpebral com ácido tricloroacético. Arq Bras Oftalmol. 1996;59(3):312-4.

12. Pedace FJ, Winkelmann RK. Xanthelasma palpebrarum. JAMA. 1965;193:893-4.

13. Santos RD. III Diretrizes Brasileiras sobre Dislipidemias e Diretriz de Prevenção da Aterosclerose do Departamento de Aterosclerose da Sociedade Brasileira de Cardiologia. Arq Bras Cardiol. 2001;77(Suppl 3):1-48.

14. Cruz AA, de Alencar VM, Falcão MF, Elias J Jr, Chahud F. Association between Erdheim-Chester disease, Hashimoto thyroiditis, and familial thrombocytopenia. Ophthal Plast Reconstr Surg. 2006;22(1):60-2.

15. Haygood LJ, Bennett JD, Brodell RT. Treatment of xanthelasma palpebrarum with bichloracetic acid. Dermatol Surg. 1998;24(9):1027-31.

16. Raulin C, Schoenermark MP, Werner S, Greve B. Xanthelasma palpebrarum: treatment with the ultrapulsed $\mathrm{CO}_{2}$ laser. Lasers Surg Med. 1999;24(2):122-7.

17. Mannino G, Papale A, De Bella F, Mollo R, Morgia P, Gabrieli CB. Use of Erbium: YAG laser in the treatment of palpebral xanthelasmas. Ophthalmic Surg Lasers. 2001;32(2):129-33.

18. Lieb WE, Klink T, Münnich S. $\left[\mathrm{CO}_{2}\right.$ and erbium YAG laser in eyelid surgery. A comparison]. Ophthalmologe. 2000;97(12):835-41. German.

19. Bagatin E, Enokihara MY, Souza PK, Macedo FS. Xantelasma: experiência no tratamento de 40 pacientes. An Bras Dermatol. 2000;75(6):705-13.

20. Parkes ML, Waller TS. Xanthelasma palpebrarum. Laryngoscope. 1984; 94(9):1238-40.

21. le Roux P. Modified blepharoplasty incisions: their use in xanthelasma. Br J Plast Surg. 1977;30(1):81-3.

22. Hosokawa K, Susuki T, Kikui TA, Tahara S. Treatment of large xanthomas by the use of blepharoplasty island musculocutaneous flaps. Ann Plast Surg. 1987; 18(3):238-40

23. Werner MS, Olson JJ, Putterman AM. Composite grafting for eyelid reconstruction. Am J Ophthalmol. 1993;116(1):11-6.

24. Mendelson BC, Masson JK. Xanthelasma: follow-up on results after surgical excision. Plast Reconstr Surg. 1976;58(5):535-8. 